Nouvelles perspectives en sciences sociales

Revue internationale de systémique complexe et d'études relationnelles

\title{
La simulation : des déplacements de notre épistémologie
}

\section{Pierre Livet}

Volume 5, numéro 2, mai 2010

Sur le thème de la simulation

URI : https://id.erudit.org/iderudit/044074ar

DOI : https://doi.org/10.7202/044074ar

Aller au sommaire du numéro

\section{Éditeur(s)}

Prise de parole

\section{ISSN}

1712-8307 (imprimé)

1918-7475 (numérique)

Découvrir la revue

\section{Citer cet article}

Livet, P. (2010). La simulation : des déplacements de notre épistémologie. Nouvelles perspectives en sciences sociales, 5(2), 51-57.

https://doi.org/10.7202/044074ar

\section{Résumé de l'article}

En sciences sociales, seules des simulations peuvent nous donner une idée de la manière dont des formes collectives résultent d'interactions complexes. Mais plus elles sont complexes - en particulier en intégrant les effets des représentations des formes collectives par les acteurs - plus les simulations peuvent diverger, d'où une indétermination. Or nous sommes plus sensibles à ce qui est pour nous reconnaissable (formes plus stables, mieux différenciées). Combiner ces deux tendances nous amène à privilégier non pas une correspondance avec une réalité elle aussi complexe, mais la capacité des simulations à permettre entre elles des comparaisons qui puissent nous offrir des moyens de les critiquer les unes par les autres. Nous conservons à l'esprit, en parallèle, les scénarios divergents qui restent les plus différentiables tout en restant attentif à des divergences d’abord négligées. 


\section{La simulation : des déplacements de notre épistémologie}

Pierre Livet

Université de Provence et Centre d'épistémologie et d'ergologie comparatives, Équipe CNRS UMR 6059

E n physique et en cosmologie, les simulations servent à nous indiquer ce que donnent comme résultats les équations de nos théories, quand celles-ci sont si complexes que nous ne pouvons trouver de solutions par simplification et démonstration, de manière analytique. Nous renonçons alors (temporairement, nous l'espérons, mais ce n'est pas assuré) à un objectif de la connaissance qui est de comprimer l'information nécessaire pour décrire la réalité. Cette compression se fait par le choix d'un nombre limité de fonctions de base qui permettent de définir le cadre des modèles physiques, modèles qui s'obtiennent à partir de la théorie en spécifiant la forme d'une fonction qui n'avait été définie que de manière générique et en fixant des paramètres ou des conditions d'approximation admissibles.

En sciences sociales, l'économie nous propose un cadre théorique d'où l'on peut tirer des modèles. Comme on peut affaiblir nombre de conditions de la théorie, la liberté de construction des modèles est très grande. Les simulations peuvent alors, dans le meilleur des cas, nous permettre de voir quels sont les résultats collectifs résultant de l'interaction d'agents supposés agir comme 
dans le modèle, et s'ils sont compatibles avec les données économétriques. Ils permettent aussi de comparer les résultats des différents modèles.

Quand on plonge des modèles de la théorie économique dans les particularités et les contraintes d'un environnement, on doit enrichir ces modèles. Là encore, les simulations jouent leur rôle d'indicateurs des résultats possibles d'interactions multiples. Mais il faut aussi tenir compte en économie - et, bien évidemment, en sociologie et en anthropologie - de ce que les acteurs se font eux-mêmes des représentations des effets collectifs. Ils observent certains effets, ils modifient leurs comportements et attendent d'autres effets, et ainsi de suite. Les simulations doivent alors tenir compte de leurs anticipations, de leur information plus ou moins précise et complète, de leurs attachements à des valeurs actives dans leur société. On complique les modèles, et plus on les complique, plus la simulation devient le seul repère pour savoir avec quels comportements collectifs les modèles sont compatibles.

\section{Sources d'indétermination}

Plus l'on s'éloigne des conditions des simulations physiques, plus les informations que l'on peut tirer des simulations deviennent délicates à interpréter, et moins elles donnent de guides clairs pour savoir comment réviser les modèles - ce qui est pourtant le but de tout le processus, si du moins nous ne nous satisfaisons pas d'avoir produit des résultats de simulation qui ont des formes similaires à des formes collectives que nous pouvons repérer. Les facteurs d'indétermination qui pèsent sur nos interprétations et nos suggestions de révision sont multiples.

Supposons que le résultat d'une simulation soit compatible avec les données. Cette compatibilité tient évidemment à des approximations - on voit des formes collectives similaires à celles qu'on observe dans la vie sociale résulter des simulations; mais cette similarité implique elle-même des interprétations. Pourquoi négliger telle différence et se focaliser sur telle similarité? Le mode de reconnaissance des formes collectives est lui-même fortement 
dépendant de nos capacités perceptives de reconnaissance de forme. On peut les encadrer par des tests statistiques, mais il reste qu'entre plusieurs résultats de simulations dont les uns nous semblent d'une irrégularité désordonnée et les autres nous proposent des formes plus connexes et plus distinctes les unes des autres, nous privilégierons les seconds, alors même que, par ailleurs, nos formalisations nous ont montré que certains chaos obéissaient à des fonctions bien repérables. Il nous faut donc analyser quelle est l'incidence des préférences propres à nos reconnaissances de formes. Mais on ne voit pas bien non plus quel sens cela aurait de défalquer les biais de ces préférences. En fait, si on reprend l'exemple du chaos, nous avons attendu que la théorie du chaos déterministe nous fournisse des modèles d'attracteurs chaotiques dont les formes globales sont plus reconnaissables pour pouvoir rapporter des données à ces formalismes. Il faudrait que nous ayons un répertoire ordonné de critères formels pour évaluer les similarités entre résultats des simulations et données effectives, au lieu d'utiliser seulement nos très (ou trop) efficaces capacités perceptives.

Comme nous avons obtenu nos modèles en affaiblissant des conditions formelles trop strictes (par exemple, nous supposons que l'ordre des préférences n'est pas complet ou n'est transitif que sur un certain nombre d'items), le nombre des résultats compatibles avec nos présupposés devient très important, puisque beaucoup de valeurs de paramètres et de spécifications de fonctions respecteront ces principes affaiblis. La méthode des économistes a été, sur ce point, de commencer par tenter de rendre compte des données avec des modèles forts, et de les affaiblir progressivement. Mais passé un certain degré d'affaiblissement, l'arbre des modifications possibles devient trop important pour que nous puissions estimer de manière précise ce qu'il est souhaitable d'affaiblir.

Une autre source d'indétermination en simulation tient aux modes d'implémentation informatique des modèles, implémentation nécessaire pour calculer les résultats collectifs d'un très grand nombre d'interactions. Les différences de programmation 
rendent délicates les comparaisons entre modèles de simulations multi-agents, mais la communauté des chercheurs en a conscience et tente de se donner des repères plus faciles à partager.

Par ailleurs, quand nous donnons aux agents des simulations des capacités " cognitives " (qui modifient leurs réactions en fonction de la manière dont ils catégorisent les résultats collectifs obtenus), nous introduisons une autre source d'indétermination. Ces catégorisations induisent des discontinuités (à l'intérieur de la catégorie, les données sont plus similaires, et entre catégories, elles le sont moins) et ces discontinuités, une fois agrégées, ont des effets collectifs qui peuvent tenir à des différences initialement faibles.

Enfin, plus les agents de nos simulations ont des capacités cognitives importantes, plus leurs décisions sont donc prises dans un aller-retour rapide avec l'observation des tendances collectives que donnent les premiers résultats des simulations et plus les résultats collectifs peuvent diverger. En soi, ces divergences ne seraient pas forcément un handicap, si elles étaient d'emblée tranchées. Le problème est que la plupart des résultats, quand on considère leur multiplicité, peuvent diverger faiblement deux à deux, alors même que des divergences initiales faibles peuvent indiquer un futur de divergences fortes [ce qu'on constate déjà dans les multiples scénarios proposés par le groupe d'experts intergouvernemental sur l'évolution du climat (GIEC) pour l'évolution du climat, et pourtant les réactions des humains n'y sont pas vraiment modélisées, sinon économiquement]. Nous ne pouvons donc nous reposer sur l'idée que seules les divergences fortes devraient nous amener à réviser nos modèles.

La nature de ces indéterminations fait qu'on ne peut pas trop espérer les dépasser. Dès lors, doit-on rester sceptique sur les simulations et déplorer leur prolifération?

\section{Réviser nos modes de révision}

Nous sommes, en fait, contraints par cette évolution épistémologique à réviser nos propres modes de révision. Popper ne nous est pas très utile : plus - ou peu-de réfutations à espérer. Lakatos 
non plus : un programme jugé dégénératif pendant un temps (par exemple, l'étude du chaos) pourra se révéler porteur ensuite. Nous devons maintenir en parallèle les différentes tentatives et les évaluer comparativement, tout en sachant que cette évaluation est elle-même révisable. Par exemple, si une simulation présente un modèle plus économe en paramètres et en fonctions que les autres et donne des résultats collectifs similaires, elle peut être préférée, mais cela se fait relativement aux autres modèles en cours. Notre raison pour la préférer, c'est qu'elle présente un avantage dans la comparaison : s'il y a plus de paramètres, il est plus facile de les bricoler pour obtenir le résultat souhaité, et donc pour brouiller la comparaison.

On voit que nous ne mettons plus au premier plan comme qualité majeure la correspondance avec les faits observés - qui ont eux-mêmes été formatés, en fait, selon des prémodélisations - mais plutôt les qualités qui facilitent l'évaluation comparative : moins de paramètres à bidouiller ou de variations sans portée sémantique claire dans la syntaxe des programmes, plus de différenciations entre résultats des simulations qui peuvent être estimées par des critères réapplicables; voire même, pour ce qui est des ajustements complexes des agents cognitifs, une focalisation sur les tendances qui sont plus basiques et moins sophistiquées, puisque les premières auront un effet de fond avec moins de bifurcations possibles, et plus facilement repérables, alors que les secondes auront des effets de variations de surface entre lesquelles il sera difficile de repérer celles qui feront boule de neige et celles qui resteront marginales.

C'est un peu réducteur, puisqu'au fond, quand nous nous focalisons sur des conditions qui rendent plus aisées et plus décisives les comparaisons et les évaluations qui en découlent, nous appliquons la tactique de l'ivrogne qui cherche sa clé sous le réverbère parce que c'est seulement là qu'il y voit. À ceci près que nous cherchons des comparaisons entre plusieurs sources de lumière ou d'information fiable (le toucher peut aussi être utile pour chercher ses clés). Et cela vaut mieux évidemment que de nous lancer dans une quête désordonnée et sans guide. 
Les simulations nous entraînent dans une épistémologie renouvelée. Au lieu de viser une confrontation directe avec la réalité par des tests expérimentaux, puis une révision des hypothèses, et ainsi de suite, nous tentons de multiples simulations en parallèle, et c'est à partir des critères que nous pouvons dégager sur leur comparaison entre elles que nous jugeons la correspondance avec les données extraites de la réalité. La similarité est alors qualifiée par l'orientation de la simulation sur tel ou tel phénomène repéré et aussi par le rapport propre au modèle entre des variations de départ et des bifurcations possibles ensuite. Nous ne pouvons plus privilégier les modèles qui pour une variation faible ne produisent que de faibles divergences, puisque la vie sociale ne leur correspond pas. Mais nous ne privilégions pas non plus ceux qui produisent des bifurcations erratiques, et nous préférons ceux qui produisent soit des bifurcations importantes, soit un retour de type ergodique au domaine de variations du départ. Nous reconnaissons que notre connaissance obéit à l'impératif de privilégier, dans un monde complexe et chaotique, ce qui reste reconnaissable, puisque c'est seulement sur ces repères que nous pouvons appuyer nos actions.

\section{Le bon timing et ses exigences}

Nous prenons alors conscience de ce qu'un tel biais nous fait prêter le flanc aux surprises d'une réalité chaotique. Nous prenons conscience aussi du fait que nous devons choisir le bon timing. Nous ne devons ni nous précipiter pour surréagir au moindre signe d'une divergence parce qu'elle pourrait se développer, ni rester fixés sur les seules variétés de trajectoires possibles que nos premiers scénarios ont programmées. Notre méthodologie pourrait comprendre les trois exigences suivantes.

1) Nous privilégierons les divergences qui se révèlent apparaître de manière repérable dans plusieurs scénarios, parce que cela revient pour ces divergences hypothétiques à avoir résisté à plusieurs tentatives de révision dans des scénarios de simulations différentes. 
2) Évidemment, nous resterons aussi réceptifs à toute donnée qui diverge de manière significative de nos scénarios, nous resterons ouverts à d'autres révisions.

3) Le plus difficile sera, en fait, d'assurer une veille concernant les évolutions que nous considérons comme marginales une fois ces deux premières exigences satisfaites, mais qui pourraient se mettre à diverger de manière significative. Or, c'est en continuant à lancer des simulations sur les effets de ces variations que nous avons des chances d'être sensibles à ces divergences possibles.

\section{Conclusion}

La simulation se révèlerait-elle alors le remède à ses propres inconvénients? Ces remèdes n'éliminent pas le mal : les biais de la simulation s'appliquent aussi aux tentatives d'utiliser des simulations pour répondre aux trois exigences que nous venons d'évoquer. Nous ne disposons pas d'autre méthode que d'appliquer notre thérapie de manière récursive : rester attentifs à nos trois exigences, réappliquer dans une seconde mise en œuvre de notre thérapie le même type de remède aux incidences que ces biais continuent d'avoir dans une première application de cette thérapie, et ainsi de suite. Il est toujours délicat de trouver un équilibre entre la focalisation sur ce que nous pouvons reconnaître - notons cependant que nos critères de reconnaissance ne restent pas figés et qu'ils évoluent en fonction de la complexification de nos connaissances - et la sensibilité à la manière dont les réalités sociales peuvent nous entraîner hors de nos schémas anticipateurs. 